\title{
Deconstructing Ambient Intelligence into Ambient Narratives: The Intelligent Shop Window
}

\author{
Mark van Doorn \\ Philips Research Laboratories \\ High Tech Campus 34 \\ 5656 AE Eindhoven, The \\ Netherlands \\ mark.van.doorn@philips.com
}

\author{
Evert van Loenen \\ Philips Research Laboratories \\ High Tech Campus 34 \\ 5656 AE Eindhoven, The \\ Netherlands \\ evert.van.loenen@philips.com
}

\author{
Arjen P. de Vries \\ Centre for Mathematics and \\ Computer Science \\ Kruislaan 413 \\ 1098 SJ, Amsterdam, The \\ Netherlands \\ arjen.de.vries@cwi.nl
}

\section{Categories and Subject Descriptors}

H.5.4 [Information Interfaces and Presentation]: Hypertext/Hypermedia

\section{Keywords}

ambient intelligence, interactive narrative, end-user programming, intelligent shop window

\begin{abstract}
Producing intelligent environments that are sensitive and responsive to the presence of users and their environment on a large scale is difficult. On one hand, it is technologically very hard to mass produce a device so smart that it is capable of generating a truly personal ambient experience for each situational context, on the other hand, tailor-made solutions are often economically infeasible. In this paper we discuss a mass customization approach towards Ambient Intelligence that tries to balance variety and cost by breaking up an intelligent environment into small, interrelated modular fragments. Through interaction with their environment users assemble these fragments in a personal story that is experienced as Ambient Intelligence. We describe the requirements derived through a series of workshops with retailers and designers and illustrate the design of the physical hypermedia model and system. Using a test case, the intelligent shop window environment, we evaluate the feasibility of the approach.
\end{abstract}

\section{INTRODUCTION}

In recent years, Ambient Intelligence has become an important area of research. In universities, companies and research institutes around the world, people are working on enabling technologies and applications. Prototype demon-

Permission to make digital or hard copies of all or part of this work for personal or classroom use is granted without fee provided that copies are not made or distributed for profit or commercial advantage and that copies bear this notice and the full citation on the first page. To copy otherwise, to republish, to post on servers or to redistribute to lists, requires prior specific permission and/or a fee.

AMBI-SYS 2008, February 11-13, Quebec, Canada Copyright (C) 2008 ICST 978-963-9799-16-5

DOI 10.4108/ICST.AMBISYS2008.2872 strations built in a user-centric way are often difficult to reuse because they are designed with one specific scenario in mind. If everything that does not directly add to the target user experience is considered a cost factor and eliminated, other important aspects such as interoperability and reuse can easily be overlooked in the design. For the further development of Ambient Intelligence this raises an important issue. How can devices work in concert in order to support our everyday activities in an intelligent, meaningful way if they are each designed for a very specific application?

Perhaps one day we will succeed in building and mass producing a device so intelligent that it is capable of generating an ambient intelligent environment that exactly fits each individual situation and even anticipates in collaboration with other devices what the next action should be, but for the near to medium term future, this seems unlikely given the state-of-the-art in artificial intelligence. At the same time it does not seem feasible to manually design and produce Ambient Intelligence applications for each person or context of use individually. In this paper we investigate the possibility of a mass customization approach towards Ambient Intelligence to combine the best of both worlds, i.e. the low-cost, standard quality of mass production and variety of traditional craftsmanship.

\section{EXPERIENCE CO-CREATION}

The goal of Ambient Intelligence is to help people in performing their daily activities better, by making these activities more convenient and enjoyable using technology. Notice the word performing in this description. The word 'performing' is so commonly used in our language that we forget that performances are pervasive to every culture. Performances are not just seen in the theatre, music, dance and performance arts in general but also in our everyday lives: We perform the role of a father or son in our private lives but maybe also that of a doctor, judge or police agent for example in our professions. Goffman [8] already recognized in the 1950s that people follow culturally specified social scripts that influence each other. Performance studies [28] takes a much wider, holistic view on what performance is and what it means to perform than traditional theatre and performance art. Viewing life as social theatre is interesting because if people behave according to social scripts, we may try to detect these scripts and connect actions to these 
events. Just as lighting and sound effects add to the overall drama of a theatre play, Ambient Intelligence may thus be applied to enhance the performance described by these social scripts.

Many social scripts are situated in private homes. The home we live in can be seen as a stage on which we perform our everyday rituals like waking up in the morning, having breakfast, bringing the children to school, going to work and so on. While we conduct these tasks we use a myriad of products (like props in a theatre play) to support our performance. The presence of social scripts is also recognized by service marketing and brand management professionals for commercial and public spaces. Pine and Gillmore [23] and Fisk and Grove [7] describe a theatre framework for service marketing, in which services are seen as performances, front-end service personnel as actors, the service setting as the stage on which the service is performed, products used in the services as props and the business process of the service as the script.

Prahalad and Ramaswamy [24] argue for a much more active role of the individual in the creation of the experience. Because experiences linger on as memories in our mind, we cannot detach ourselves from our experiences. Experiences include us and we are included in our experiences. Our experiences define to a large extent who we are. This implies that the production and consumption of experiences cannot be separated and that strictly speaking companies cannot produce experiences. What organizations can do, is provide an environment that supports the formation of experiences. The personal meaning derived from this co-creation process is what determines the value for the individual.

\section{AMBIENT NARRATIVES}

To understand how Ambient Intelligence comes into the picture, it is helpful to analyze how personal experiences and meaning are formed in co-creation environments from a linguistic, semiotical point of view. This step is necessary if we want to arrive at a suitable machine readable representation.

In semiotics (see e.g. [3] for a basic introduction), a sign can be understood as a unit of meaning, and include words, images, gestures, scents, tastes, sounds or any other way in which information can be communicated as a message. When we watch a movie or read a book, we decode the signs in the narrative written by the author. The experience leaves an impression, a memory in our mind in a way we cannot control: We can look back at the experience when it has happened, but we cannot change the effect it had on us. Furthermore, the interpretation of the experience, the meaning we assign to the experience, depends not only on the signs themselves, but also on the situational and cultural context. In a similar way, when we experience a product, we decode the signs encoded in the product by its manufacturer. This not only includes perceptual attributes like the look-and-feel of the product or its user interface but also intangible, invisible attributes such as the brand image. With traditional media like film, newspapers and television, but also in case of non-interactive products and services, the author/producer has composed a linear narrative that is revealed through experience with the product. Although the experience can be highly immersive, the plot of the story is not under control of the reader: When we watch a movie, read a novel or ride a rollercoaster, the sequence of signs is fixed.
Interactive narratives such as hypertext novels and computer games enable readers/players to control, change or otherwise affect the plot of the narrative by interaction [18]. Depending on the choices readers make, a different story is conveyed. In a similar way, buildings, landscapes and cities give people choices that affect the outcome of the experience. The author of these environments has created a structure where readers can choose between different, carefully predefined, interrelated 'signs'. Murray proposes three aesthetic categories for the analysis of virtual interactive story experiences: immersion, agency, and transformation [20]. Virtual environments aim for high levels of immersion, agency and transformation to create natural, realistic experiences. Because mixed reality environments are a combination of reality and virtuality, we expect the same for ambient intelligent environments.

Experience co-creation environments differ from interactive environments in the sense that they enable readers to not just choose between different signs but also change the existing sign set by adding, removing or changing signs. Readers in the process effectively become writers, producers of signs themselves. The action of choosing a narrative sequence in an interactive narrative is a unit of meaning and therefore can be considered as a sign writing action. However, all possible actions and outcomes must be thought of in advance by the author, the designer of the interactive narrative. The latter is not necessary in co-created, emerging narratives.

It is now possible to position Ambient Intelligence into this picture. If we break down an Ambient Intelligence environment into a large number of small, interrelated, modular fragments consisting of signs (or sign generators) addressing different senses called an ambient narrative. By performing in the environment, the reader of the ambient narrative implicitly sequences these fragments and assembles his own story, his own experience. If the ambient narrative is designed well by the author of the ambient intelligent environment, this story is perceived as Ambient Intelligence. The narrative structure is not disconnected from the environment in which we live, as in virtual reality, but an integral part of it: The artificial and real signs are jointly experienced and must be therefore be written, produced in an holistic way. To support experience co-creation, it should be easy for readers of the ambient narrative to add, remove or modify these fragments.

\section{RETAIL WORKSHOPS}

To derive concrete requirements for a language to describe ambient narratives in a machine readable format and to come up with an underlying system architecture, we performed a series of workshops and interviews with retailers, retail consultants and designers. There are a number of reasons we choose the retail domain. First, shops and brands have to differentiate themselves from the competition. Stores must have their own unique identity. Second, store owners want to change their collection frequently, at least four times per year but preferrably more.

In 13 sessions we spoke to 17 people with various backgrounds (retail management, hotel management, industrial design), levels of expertise (2-20 years) and positions (professional designers, store owners, independent consultants and executives) in the Netherlands and Finland and asked them about their current way of working with regard to design- 


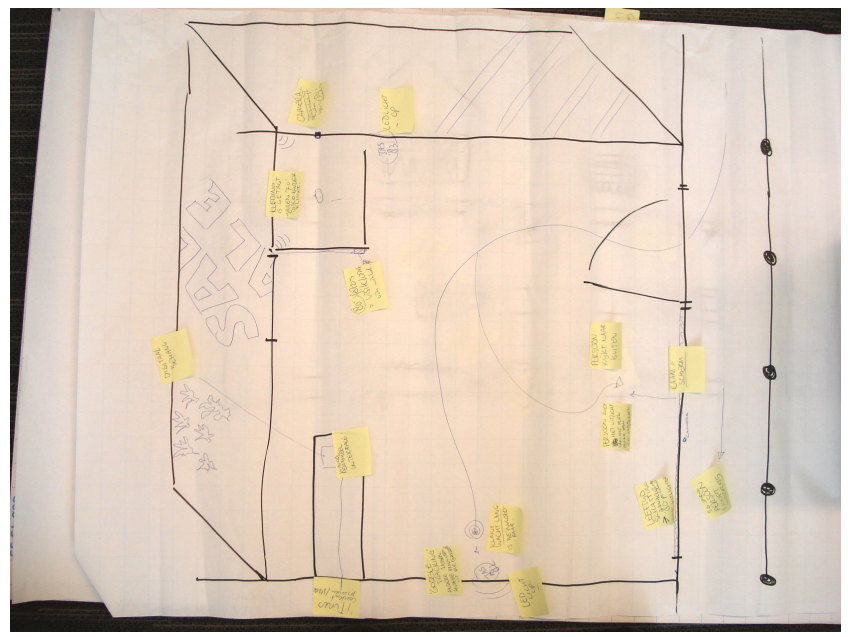

Figure 1: One of the ambient narrative examples created during the workshops

ing, configuring and installing interactive ambient effects, media and applications in retail. Most retailers and consultants were positive about the use of ambient media in retail spaces and believed it could become a strong differentiator. But, many remarked that the use of interactive media and ambient effects should not be overdone or distract. Ambient media should fit into the overall retail concept as a whole and add meaning to it in terms of experience or convenience. Both the designers and retailers commented that Ambient Intelligence did not play an important role in their current practice. The use of ambient media today is limited to lighting, audiovisual media (e.g. projections) and interactive applications for providing information. However, most participants said they would very much welcome more flexibility in adapting the perception of the store.

We felt we could not ask people directly to specify realistic ambient narratives because the abstract concept of ambient narratives is difficult to convey to end-users. Therefore we took an indirect approach. First, we asked people to imagine a real store or store concept and write down a number of use-case scenarios from the point of view of the individual customer visiting this store. If they had difficulty in coming up with scenarios we asked them what would happen if somebody would walk by the store, stood still in front of the shop window display, entered inside, browsed through the store, etc. to stimulate their imagination or direct their creativity. After this step we presented them with the service marketing framework of Fisk and Grove [7] (see section 1) and asked them to decompose the use-case scenarios into theatrical scenes each consisting of an action and a situation description and write them down on a post-it they had to position on a large piece of paper that represented the store layout.

\section{Example}

Figure 1 shows one of the 13 ambient narratives we collected. The participant came up with a second-hand article shop concept where people can buy second-hand objects such as clothes, books, radios, clocks, furniture. The post-its represent the scenes, the position on the piece of paper indicates where the scene is located in space. For example, one post- it near the dressing room says kleding is getagt, jaren 70 disco muziek klinkt (clothing is tagged, disco music from the $70 \mathrm{~s}$ is played). When a tagged piece of clothing is inside the dressing room, music starts to play in the dressing room that matches the style and time of the clothes was the underlying idea here. Another example consisted of two post-its to represent a single scene in front of the shop window, outside of the store: 20 jarige loopt voorbij (20-year old walks by) and leeftijdschatting - aanbeveling 80 product d.m.v. uitlichting (age is estimated and 80s-product is highlighted).

\section{Analysis}

The series of workshops and interviews resulted in dozens of use-case scenarios and hundreds of post-its with scene descriptions. The store layouts on paper, the writing on the post-its and the position of the post-its on the pieces of paper contain interesting material for further analysis. The participants with a design background were generally faster at generating use-case scenarios. The consultants and retailers were much more thinking in terms of what the store should communicate to different potential customers, their creativity expressed itself at a more abstract level. All the participants could find themselves thinking in the service as theatre model, most people were focussed on the action description and less on the exact specification of the situation or on dependencies with other scenes. This may indicate that people want to design and specify in an action-driven way. We did not structure or guide this process too much because we also wanted to discover how people would like to articulate the scenes.

Because the retail workshops came up with all different store concepts ranging from e.g. a clothing store, fish outlet center, hairdresser salon, computer store to jewelry shop we believe the data we collected is not just applicable for a single type of retail environment. The retail scenarios refer to traditional media objects (i.e., text, sound, images and video), ambient effects and/or one or more application objects that start and stop at different times on different devices.

Based on the retail workshops and a complementary literature study (see also [30]) we found the following context parameters and types of actuators, applications and sensors. The frequently occurring context parameters (information derived from sensors) are the relative or absolute position of a person with regard to an object (e.g. device or item), the identity (profile) of an object or person, the relative or absolute position of an object in the store, relation between a person and an object (e.g. person touches device), orientation of a person, activity of a person (e.g. idle, walking, sitting), capabilities of a device, the date and time, light or temperature outside. Dependencies upon other scenes (e.g. scene A should not start unless scene B is active) were seldom mentioned. One reason could be that people want only simple reactive behavior that can be quickly understood, another reason could be that people simply did not think of this.

Participants were at least as interested in the appearance and perception of the retail environment as in the interactive possibilities, if not more. In terms of modalities, the choice of media primarily depends on the specific retail setting, but in general (colored) lighting, images, videos and text were considered important media to communicate with potential customers. Music was often regarded as distracting 
for other customers or too dependent on taste, yet in specific situations background ambient sound or directed speech was seen as adding value. Scent was not often mentioned but considered important (e.g. bakery, fish shop) although not something people would want to artificially create in most cases. In some cases (e.g. high end fashion stores that emphasize product authenticity), even non-interactive colored lighting or video projections were however seen as undesirable. No specific requirements could be found with regard to the applications that could be triggered in response to user behaviour. They varied from a clothing catalogue to an application for letting end users design jewelry.

\section{SYSTEM DESIGN}

As demonstrated in [31], ambient narratives are naturally represented in a hypertext model supported by an ambient narrative engine, to sequence ambient narrative fragments and create the ambient intelligence experience. First, the language model is discussed, then we describe the ambient narrative engine in detail.

\section{Language Model}

The hypertext language to represent ambient narratives supports the following features:

- Action customization: The presentation can be personalized for a particular user or context situation, unknown at the time of authoring the presentation description.

- Situational actions: Contextual restrictions (e.g. spatial and temporal constraints) to define context-aware behavior

- Contextual linking: Link traversal can be initiated by any change in context situation (e.g. person moves in/out a particular zone).

- Session state reasoning: The activation of a presentation description is not only dependent on contextual information including user input, but also on past (e.g. user preferences) and current system state (other ambient presentations active, user goals).

- Timing and synchronization of remote procedure calls: An ambient presentation description can consist of traditional media objects, ambient effects and/or one or more application objects that start and stop at different times on different devices.

- Concurrent browsing: In realistic ambient spaces multiple actors can be involved in multiple performances.

Figure 2 shows the UML static structure model that contains the main language constructs. The nodes of an ambient narrative are called beats. The term beat originates from theater play and film script-writing and is defined [17] as a change in the behavior of a character. The beat is the smallest unit within a scene, that in turn represents a greater change in behavior. By performing in their environment, i.e. by doing things, people cause beats to be sequenced into a coherent story. This beat (sequencing) approach is adopted from interactive drama e.g. $[5,16,15]$.

Each beat consists of a precondition (pre) and action section (situational actions). The precondition section describes the restrictions on the context in terms of stage (location), performance (activity), actor (person), prop (device or tangible object) and script (session, story state) constraints. The element's attributes (not shown) allow the author to specify more detailed constraints such as the name of the actor, the type of device, the relative position of a device with regard to an actor, the kind of activity, the existence or non-existence of a story value etc. In total we identified 92 unique context parameters, see [30]. Only after a beat is ready for activation and all its preconditions have been satisfied, can the action part be executed.

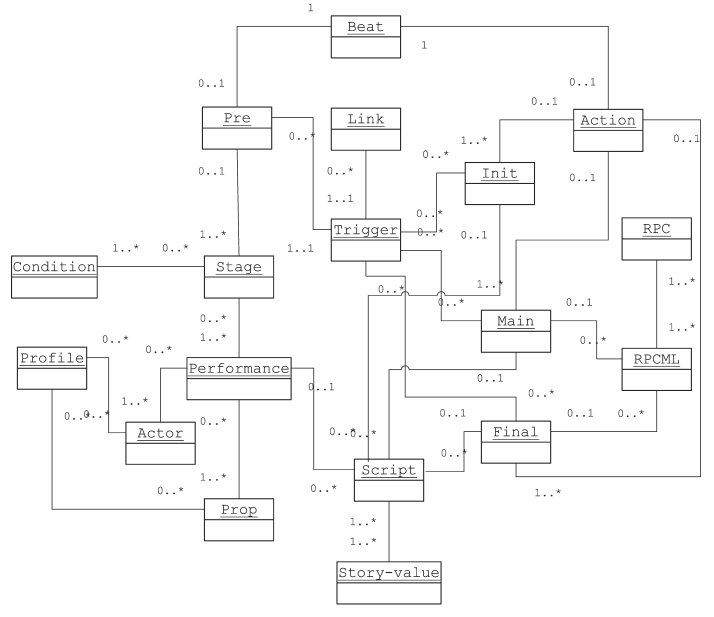

Figure 2: UML static structure model of ambient narrative language

The action section describes what happens when the beat is activated and consists of an init, main and final section. The init section describes what happens when the beat is started (initalization), the final section is called when the beat is stopped (finalization). The main section contains the actual ambient presentation, the actions defined by the beat. It defines the timing and synchronization of media objects (timeline starts when the beat is activated), ambient effects, or, in general, remote method invocations on application objects. Actions are specified as a remote procedure script (RPCML element) that contains zero or more timesynchronized simple remote procedure calls (i.e., no return values and no complex data types). A remote procedure call consists of its begin time and duration, and the parameter value (string) that is passed to the remote object. Actions can be customized by expressing the parameter value as an XPath query [33] (evaluated on the context model of the ambient narrative engine, explained below).

Preconditions and actions can be extended with script elements. The script element can be applied to set or remove story values before, during or after a beat is activated. This allows the author to set variables in the action element, that can be checked in the precondition section (session state reasoning).

Finally, actions can also contain trigger elements. Triggers enable the author to specify context-aware links (contextual linking), that can be set or retracted at any time. This ensures that the total list of context-aware links the engine must check is manageable. The precondition of a trigger 
defines the restrictions on the context that must hold before the link is traversed. A trigger's link element allows the author to specify which beats should be added to and/or removed from the active set through an XPath query on the beat database. Combined with the possibility to manage the addition/retraction of remote procedure scripts (attribute of the RPCML element) this facilitates concurrent browsing. Additionally, queries in the link element provide the option for run-time personalization of the navigation structure.

The preconditions part is similar to the selection knowledge stage in Mateas' Fa cade interactive drama architecture [16] that also uses tests on story memory values (and prior probability on beats). The action stage in Facade roughly corresponds to our action and post-conditions parts in the beat document. Magerko's IDA architecture [15] represents plots at the scene level and consists of five stages: initial state, required events, background knowledge, content constraints and temporal constraints. The initial state sets up the scene and may be similar to the initialization part in our action stage. The required events and background knowledge are comparable with our preconditions stage, while the content constraints that limit the binding of the variables used in the required events are similar to the embedded queries in the main action description part of our ambient narrative beat documents.

\section{Beat Sequencing}

The main task of the ambient narrative engine is to determine given the current context (including user feedback), state, and available plot material which beats must activated and/or deactivated. Figure 3 shows a flow chart of the ambient narrative engine, the central component in the system architecture.

The engine continuously listens for (filtered) context changes. If an event is received, the context model is updated. The context model used by the ambient narrative engine is similar to the preconditions area of the language model in Figure 2, except that the context model has multiple stages and absolute values instead of intervals or relative values. When the context model is updated, the existing set of active beats is checked first, whether one or more beats have become invalid because the context restrictions no longer hold, the final sections of these beats are inspected and if needed the story memory, active triggers and/or scripts meant for the rendering platform updated. If the story memory has changed, the active beats must be rechecked. If there are no invalid active beats and the story memory has not changed, the list of triggers is checked. If the preconditions of a trigger (that is not yet active) are satisfied, the corresponding link, query is executed. Depending on the type of query, zero or more beats may have to be added and/or zero or more beats should be removed. Beats that need to be added are finalized first (queries in remote procedure call descriptions resolved) if necessary. After this step the active beat set, story memory, trigger list and scripts are updated. When no more triggers are activated and the story memory is unchanged, the remote procedure call scripts are forwarded to the rendering platform that will instruct the devices to change state.

In theory, race conditions can occur when the active beat set is modified. This happens for example when multiple beats become active at the same time and set story values that are checked by others in this set; the order of activation

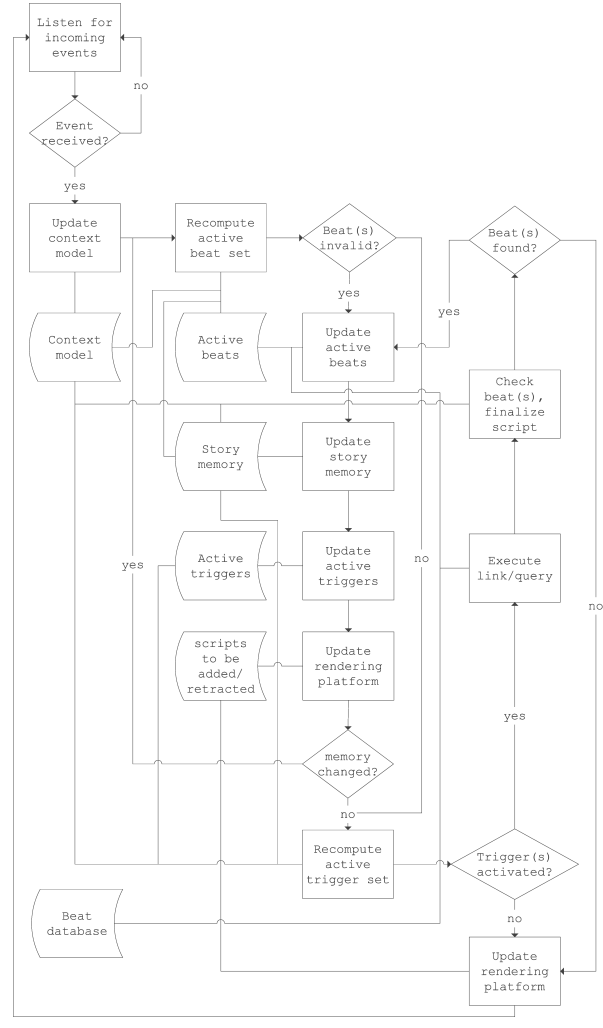

Figure 3: Flow chart ambient narrative engine

then determines the outcome. Authors can prevent these situations by specifying more detailed preconditions, such that less beats become active at the same time. We do not intend to address this potential problem in the ambient narrative engine, but leave it to the authoring environment to detect and report such situations. The individual beats in the example ambient narratives we derived from the user studies were very independent, so in practice it may not be a real issue.

The algorithm to check if a particular beat matches with the current context and story state boils down to matching document trees (not shown here). If one precondition (leaf) is not matched, the result of the comparison is false. By doing the more simple checks first, the process can be optimized.

\section{INTELLIGENT SHOP WINDOW CASE}

To evaluate the feasibility of our approach we picked an existing user-centric designed, monolithic intelligent environment, the intelligent shop window (ISW) in ShopLab [6], to see if we could rewrite this interactive installation as an ambient narrative in our system design. The reason for choosing this particular case is that it is a realistic example, confirmed by the many positive reactions of visitors (users as well as retailers) to the intelligent shop window set-up in ShopLab and the fact that similar concepts also came up during the retail workshops.

From earlier interviews with shop owners and retail experts we learned that shop windows are important marketing tools, with three main functions: 1) to express the type and style of the shop, 2) to create the appropriate atmo- 


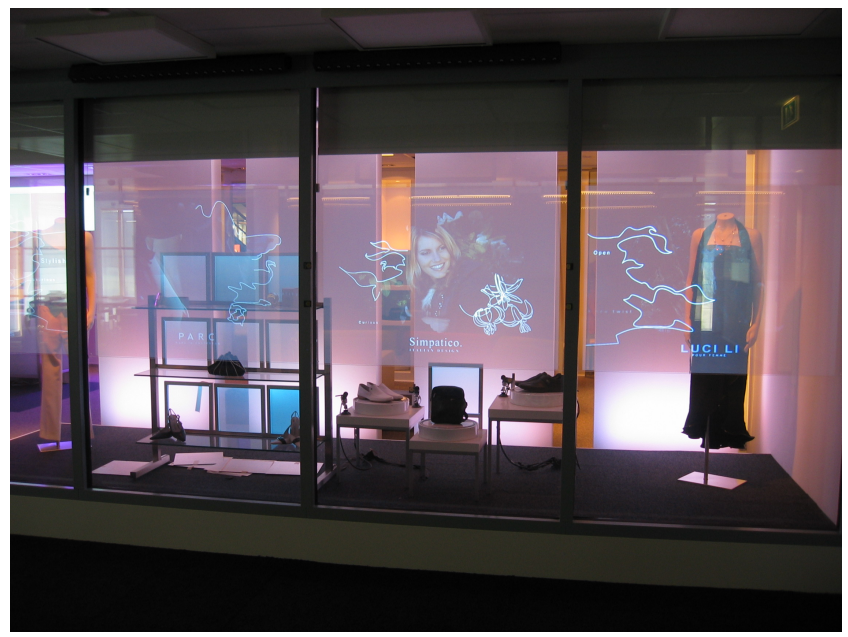

Figure 4: Intelligent shop window set-up in ShopLab

sphere and attract people and 3) to inform people about the products available and their functions. The intelligent shop window concept was developed to enhance these three functions using technology. It is related to the public ambient display that reacts to the presence and distance of people described in [32], but using a transparent display gives an even stronger sense of augmented reality. When nobody is close, the entire shop window front is used for example to project images on the transparent shop window displays that express the type and style of the shop (signage mode) as shown in Figure 4. When a person stands still in front of one the windows, the shop window can be used to get additional information about the products in the store (interaction mode). Simply by looking at the products in the shop window or touching the screen, users can bring up extra product information.

\section{Rewriting}

A number of steps were taken to rewrite the original monolithic application: Decomposing the application intelligence in fragments (writing the ambient narrative), writing the required (reusable) I/O components and connecting them to the system, and testing the new modular version on its real-time performance and functionality. The refactored intelligent shop window environment was intensively shown during a period of two weeks to hundreds of internal and external visitors, without noticeable performance difference between the modular and the monolithic versions.

Figure 5 shows the intelligent shop window set-up. The ambient narrative engine discussed in section 5 forms the central component. The rendering engine controls timing and synchronization of media, ambient effects and applications over the rendering devices. The rendering devices and applications control how the commands from the rendering platform are executed. They register themselves to the rendering platform and the sensor network. In total 24 rendering devices are used: Four shop window applications written in Java with a Flash UI running on four different PCs connected to a transparant holographic foil, four DMX LED wallwashers, ten DMX LED tiles, four directional audio devices and two spot lights. The context server collects, filters and aggregates sensor data for the ambient narrative engine. A pressure sensitive floor mat (Tekscan) and a tagbased UWB location system (Ubisense) provide sensor input (position and identification of people, objects) to the context server. Notice that the touch screen sensors by-pass the ambient narrative engine and are fed directly into the ISW applications (these are viewed as stand-alone applications, with their own I/O).

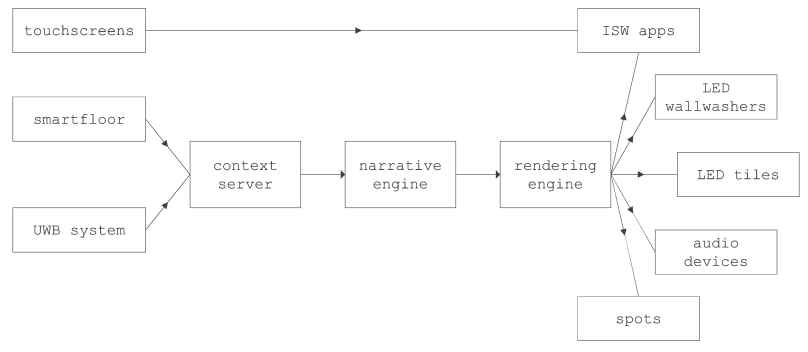

Figure 5: System architecture

The intelligent shop window environment with four windows can be described by an ambient narrative consisting of ten beats: One 'initialization' beat with an empty precondition section (bootstrap) and an init section containing nine triggers, one for the 'signage' beat, four for the 'interaction' mode on the shop windows and four for the 'blank' modes (when somebody is close to a shop window, all other screens where nobody is standing in front of should be transparent. The preconditions in the attraction beat test on the non-existence of interaction beats (modelled as story values). The preconditions in the blank beats test on the nonexistence of a customer actor. The interaction beat for the first shop window is listed below.

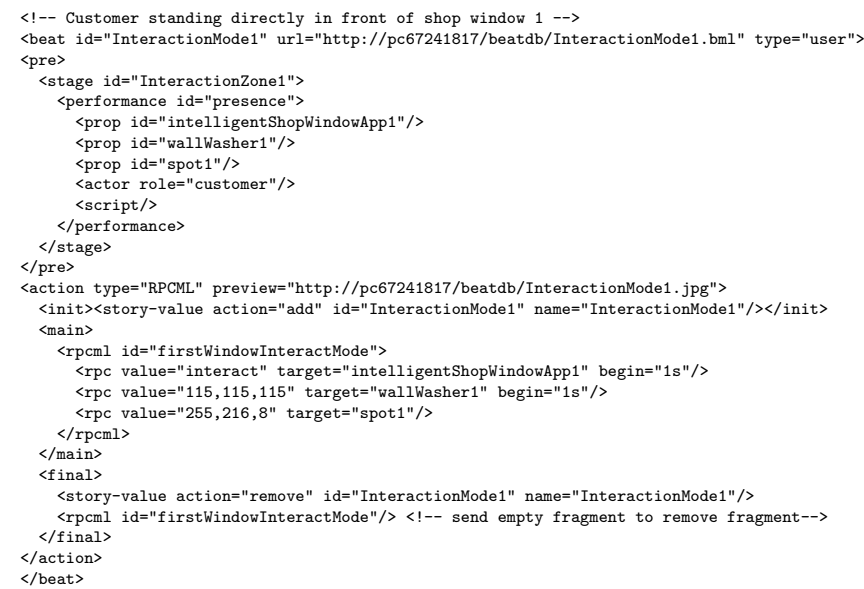

\section{RELATED WORK}

Our approach is based on the idea that ambient intelligence can be viewed as a 'story' that emerges from user interaction with a mixed-reality environment. Using hypertext as a medium for storytelling traces back before the birth of the World Wide Web. Michael Joyce's "Afternoon, a story" (1987) and Stuart Moulthrop's "Victory Garden" (1991) are some early examples of text-based hypertext novels. Later, hypermedia narratives were also demonstrated, HyperSpeech [1], HyperCafe [27] and HyperDoc [19] being some of the examples. More recent publications have expanded models and techniques into mixed reality [29, 26], 
physical $[9,11]$ and mobile environments [22], the application often having a clear narrative component (e.g. locationbased gaming, museum tours).

Ambient intelligence environments like the intelligent shop window environment differ from augmented reality environments and mobile context-aware applications in the sense that the interaction is typically distributed over multiple devices. StoryML [12] introduces an adaptive architecture for presenting interactive timed media onto distributed networked devices. In StoryML, devices are represented by 'interactors'. In a story script the author defines different tasks to these agents. However, in most ambient environments the behavior of people is difficult to predict and subject to change, which makes it hard to write such a story script. For this reason we chose to decompose the ambient environment into smaller fragments that are sequenced by a central component into a coherent story. This approach was inspired by interactive drama systems e.g. [5, 16, 15] that view interactive storytelling as an action selection problem where a central component or drama manager has to determine which story fragments must be activated or deactived based on the user's input and the story state. The Façade architecture [16] for example consists of characters written in a reactive-planning language $\mathrm{ABL}$, a drama manager that sequences dramatic fragments, a forward-chaining rule system for understanding and interpreting natural language and gestural input from the player, and an animation engine that performs real-time non-photorealistic rendering, spoken dialog, music and sound, driven by and providing sensing data to the ABL behaviors. Although we believe ambient intelligent environments and graphical multi-player computer games have certain things in common, the underlying application domains are clearly different and therefore the requirements placed upon the system.

The idea of deconstructing application intelligence into smaller entities or components has also been described in ubiquitous computing literature. The SpeakEasy [21], Jigsaw [25] and iStuff [2] systems for example follow a system design philosophy that starts with individual entitities that can be combined and recombined in multiple ways, under different circumstances and for different purposes. Newman et al. [21] describe a user interface (SpeakEasy browser) that enables end users to discover, control and connect to any available component on the network (direct connection mode of operation) or select a task-oriented template that they can fill in. The browser also supports people to modify, delete or add new task-templates. Rodden et al. [25] use a metaphor of puzzle pieces that end users can pick up and assemble in an editor to connect a pushbutton being used to signal a webcam to take a picture for example. Ballagas et al. [2] describe a central server process (Event Heap) that receives events from physical wireless input and output devices (iStuff components) in the room and redistributes them to the appropriate recipients (applications). Through a so-called PatchPanel end users can create event mappings. Coutaz [4] gives an overview of meta-user interfaces for ambient spaces. Rather than following a device-centric view where users can discover, control and connect components, we have chosen for a performative view where users can easily specify situated actions as argued in section 2 and by Jacucci [13].

Most of the research in the area of interactive ambient spaces has concentrated on the home domain. Ubiquitous computing has been proposed for interactive mobile marketing, see e.g. $[14,10]$ but this is often focussed on contextaware mobile devices.

\section{CONCLUSIONS AND FUTURE WORK}

We have shown how a realistic Ambient Intelligence application, an intelligent shop window environment that reacts to the presence and activity of people in its surroundings, can be written down in a general ambient hypermedia language and supported by an underlying platform. By separating ambience from intelligence and breaking the intelligence up into a number of small, interconnected modular fragments that are sequenced based on user feedback and context information, we demonstrate a mass customization approach towards Ambient Intelligence where intelligence is hosted in the network and ambience created locally.

From the retail workshops and the interviews with retailers and designers that visited ShopLab for the intelligent shop window we learned that the ability to customize the environment is key. Each store is unique and changes frequently (e.g., whenever a new collection arrives). This implies that the store's ambient narrative cannot be designed in advance by the producer. Instead of designing a specific application for the user, the producer needs to create an environment where end-users, in this case retailers and retail designers, can program their own intelligent shop window environment in an easy manner. Currently, we are therefore in the process of setting up a new series of experiments to find out what people think of different end user programming environments and designing a user interface for authoring ambient narratives such as the intelligent shop window environment.

\section{REFERENCES}

[1] B. Arons. Hyperspeech: Navigating in Speech-Only Hypermedia. In UK Conference on Hypertext, pages 133-146, 1991.

[2] R. Ballagas, M. Ringel, M. Stone, and J. Borchers. iStuff: A Physical User Interface Toolkit for Ubiquitous Computing Environments. In Proceedings of the ACM CHI 2003 Conference on Human Factors in Computing Systems, pages 537-544, Ft. Lauderdale, Florida, USA, April 2003.

[3] D. Chandler. Semiotics: The Basics. Routledge, 2001.

[4] J. Coutaz. Meta-User Interfaces for Ambient Spaces. In Proceedings of Tamodia '06, 2006.

[5] G. Davenport and M. Murtaugh. Automatist Storyteller Systems and the Shifting Sands of Story . IBM Systems Journal, 36(3):446-456, 1997.

[6] E. van Loenen, T. Lashina, M. van Doorn. Ambient Lifestyle: From Concept to Experience, chapter Interactive Shop Windows. Amsterdam:Bis Publishers, 2006.

[7] R. Fisk and S. Grove. The Service Experience as Theater. Advances in Consumer Research, 19:455-461, 1992.

[8] E. Goffman. The Presentation of Self in Everyday Life. Doubleday: Garden City, 1959.

[9] K. Gronbaek, P. Orbaek, J. F. Kristensen, and M. Eriksen. Physical Hypermedia: Augmenting Physical Material with Hypermedia Structures. Hypermedia, 9(1):5-34, 2003. 
[10] S.-Y. Han, M.-K. Cho, and M.-K. Choi. Ubitem: A Framework for Interactive Marketing in Location-based Gaming Environment. In International Conference on Mobile Business, pages 103-108, 2005.

[11] F. Hansen, N. Bouvin, B. Christensen, K. Gronbaek, T. Pedersen, and J. Gagach. Integrating the Web and the World: Contextual Trails on the Move. In Proceedings of the Fifteenth ACM conference on Hypertext and Hypermedia, pages 98-107, Santa Cruz, USA, 2004.

[12] J. Hu and L. Feijs. An Adaptive Architecture for Presenting Interactive Media onto Distributed Interfaces. In The 21st IASTED International Conference on Applied Informatics (AI 2003), pages 899-904. ACTA Press, 2003

[13] C. Jacucci, G. Jacucci, I. Wagner, and T. Psik. A Manifesto for the Performative Development of Ubiquitous Media. In Critical Computing, pages 19-28, 2005.

[14] S. Kurkovsky and K. Harihar. Using Ubiquitous Computing in Interactive Mobile Marketing. Personal Ubiquitous Computing, 10(4):227-240, 2006.

[15] B. Magerko. Story Representation and Interactive Drama. In Proceedings of AIIDE, Los Angeles, USA, 2005.

[16] M. Mateas and A. Stern. Structuring Content in the Facade Interactive Drama Architecture. In AAIDE, Los Angeles, USA, 2005.

[17] R. McKee. Story: Substance, Structure, Style and The Principles of Screenwriting. Regan Books, 1997.

[18] M. Meadows. Pause and Effect: The Art of Interactive Narrative. New Riders Publishers, 2002.

[19] D. Millard and e.a. Hyperdoc: An Adaptive Narrative System for Dynamic Multimedia Presentations. In Proceedings of the 14 th Conference on Hypertext and Hypermedia, 2003.

[20] J. Murray. Hamlet on the Holodeck: The Future of Narrative in Cyberspace. MIT Press, 1998.

[21] M. Newman, J. Sedivy, C. Neuwirth, W. Edwards, J. Hong, S. Izadi, K. Marcelo, and T. Smith. Designing for Serendipity: Supporting End-user Configuration of Ubiquitous Computing Environments. In Symposium on Designing Interactive Systems, pages 147-156, 2002.

[22] D. Petrelli and E. Not. User-centred Design of Flexible Hypermedia for a Mobile Guide: Reflections on the HyperAudio Experience. User Model. User-Adapt. Interact., 16(1):85-86, 2006.

[23] J. Pine and J. Gillmore. The Experience Economy. Harvard Business School Press, 1999.

[24] C. Prahalad and V. Ramaswamy. The Future of Competition: Co-creating Unique Value With Customers. Harvard Business School Press, 2004.

[25] T. Rodden, A. Crabtree, T. Hemmings, B. Koleva, J. Humble, K.-P. Akesson, and P. Hansson. Configuring the Ubiquitous Home. In COOP, pages 227-242, 2004.

[26] L. Romero and N. Correia. HyperReal: A Hypermedia Model for Mixed Reality. In Proceedings of the 14th ACM Conference on Hypertext and Hypermedia, Nottingham, UK, 2003. ACM.
[27] N. Sawhney, D. Balcom, and I. E. Smith. HyperCafe: Narrative and Aesthetic Properties of Hypervideo. In UK Conference on Hypertext, pages 1-10, 1996.

[28] R. Schechner. Performance Theory. Routledge: New York, 1977.

[29] P. Sinclair, K. Martinez, D. Millard, and M. Weal. Links in the Palm of your Hand: Tangible Hypermedia using Augmented Reality. In Proceedings of the 13th Conference on Hypertext and Hypermedia, Maryland, USA, 2002.

[30] H. van den Heuvel. Context-awareness in Ambient Intelligence Surroundings. Master's thesis, Technical University of Eindhoven, 2006.

[31] M. van Doorn and A. de Vries. Co-creation in Ambient Narratives. In Proceedings of Ambient Intelligence for Everyday Life (Lecture Notes on Artificial Intelligence 3964), 2005.

[32] D. Vogel and R. Balakrishnan. Interactive Public Ambient Displays. In Proceedings of ACM UIST, pages 137-146, 2004.

[33] XML Path Language (XPath) W3C standard. http://www.w3.org/TR/XPath/. 